\title{
Büyükyayla (Eskişehir) Florasına Katkılar
}

\author{
Süleyman ARI, Atilla OCAK, Derviş ÖZTÜRK \\ Afyon Kocatepe Üniversitesi, Fen-Bilimleri Enstitüsü, Biyoloji Bölümü, 03200 Afyonkarahisar. \\ Eskişehir Osmangazi Üniversitesi, Fen Edebiyat Fakültesi, Biyoloji Bölümü, Eskişehir. \\ e-posta: sylmanari@hotmail.com,aocak@ogu.edu.tr
}

Geliş Tarihi:15.03.2013; Kabul Tarihi:03.06.2013

Anahtar kelimeler

Flora; Büyükyayla;

Biyoçeşitlilik; Türkiye;

(B3:Eskişehir)

\begin{abstract}
Özet
Bu çalışmada araştırma alanı olarak seçilen Büyükyayla, Eskişehir ilinin güneybatısında yer almaktadır.

Araştırma alanında 55 bitki familyasına ait, 170 cins ve 230 tür ve tür altı takson belirlenmiştir. Belirlenen taksonlar içinde en fazla orana \% 11 ile Asteraceae familyası sahiptir. Bu familyayı, \% 9,8 ile Fabaceae familyası ve \% 9 ile Liliaceae familyası izlemektedir. Florayı oluşturan türlerin fitocoğrafik bölgelere göre dağılımlarına bakıldığında, Mediterranean elementlerinin \% 6,96 İran-Turan elementlerinin \% 4,78 Euro-Siberian elementlerinin \% 5,2 lik bir orana sahip olduğu görülmektedir. Çalışma alanındaki endemik tür ve türaltı takson sayısı 16 olup endemizm oranı \% 6,96'dır. Alan vejetasyonunun büyük bir kısmını orman, step, sub-alpin çayır ve nemli dere vejetasyonu oluşturmaktadır.
\end{abstract}

\section{Contributions to the Flora of Büyükyayla (Eskişehir)}

\begin{abstract}
In this study, the choosen research area of the floristic composition of Büyükyayla (B3:Eskisehir) is around the southwest side of the Eskişehir. In the research are 230 species and subspecies taxa which

Key words

Flora; Büyükyayla;

Biodiversity; Turkey;

(B3:Eskişehir) the determined taxon. After this familia there are Fabaceae family with the rate of $9,8 \%$ and Liliaceae family with the rate of $9 \%$. When we check the distributions of the species, composed of flora according to phtogeographical areas; we see that Mediterrane Elements with the rate of $6,96 \%$ is the first, it is followed by İrano- Turanian Elements with the rate of 4,78\% and Euro-Siberian Elements with the rate of $5,2 \%$. The number of the endemic species and subspecies taxon are 16 and the rate of the endemism is 6,96. Area vegetation is composed of largely by forest, steppe, sub-alpin meadow and stream vejetations.
\end{abstract}

C Afyon Kocatepe Üniversitesi

\section{Giriş}

Araştırma alanımız P. H. Davis'in (1965) grid sistemine göre göre B3 karesi içinde kalmaktadır. Büyükyayla; Eskişehir Seyitgazi-Kırka bölgesindedir. Eskişehir'in güneybatısında yer alır. Araştırma alanın en yüksek noktası 1450 m' dir. Coğrafik konum olarak; enlem 39.180 ve boylam; 30.55 koordinatlarındadır.

Eskişehir Meteoroloji İstasyonundan alınan ölçümler sonucu yağış rejimi K.I.S.Y. (Merkezi Akdeniz Yağış Rejimi Tipi) ve yağış rejimi tipinin de, Doğu Akdeniz İklimi I. tipi olarak bulunmuştur.

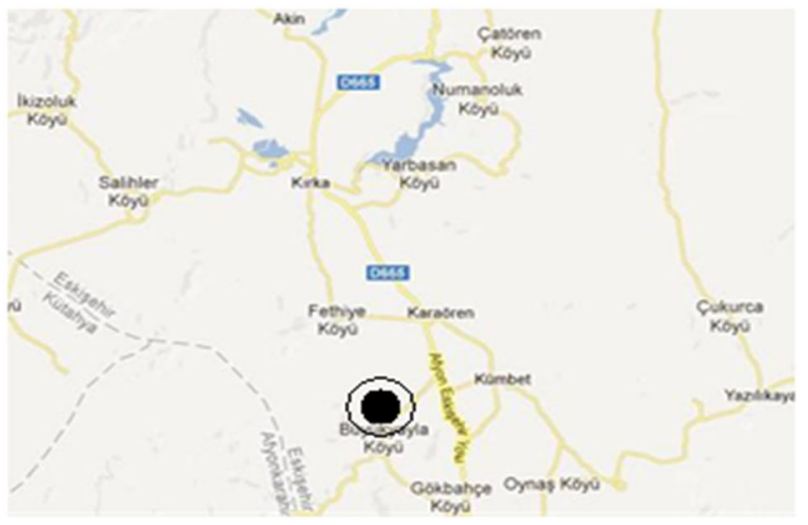

Şekil 1. Büyükyayla (Eskişehir) Topografik haritası. 


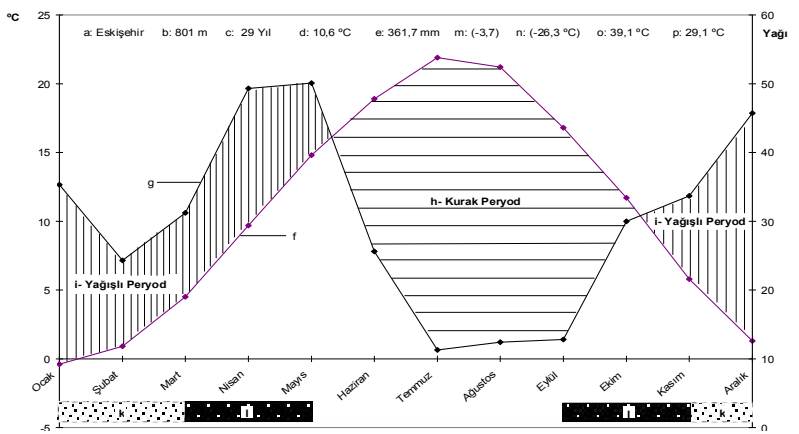

Şekil 2. Eskişehir ilinin iklim diyagramı (Walter 1965’ e göre).

$\mathrm{Q}=32-63$ arası değerleri itibariyle Yarı Kurak Akdeniz biyoiklim katına girmektedir (Akman 1990).

Tablo 1. Emberger Metoduna Göre Araştırma Alanının Biyoiklim Katı, (1975-2004).

\begin{tabular}{|l|l|l|l|l|l|l|l|l|}
\hline istasyon & $\begin{array}{l}\text { Rakım } \\
(\mathbf{m})\end{array}$ & $\mathbf{P}(\mathbf{m m})$ & $\mathbf{M}$ & $\mathbf{m}$ & $\mathbf{Q}$ & $\begin{array}{l}\mathbf{P E} \\
(\mathrm{mm})\end{array}$ & $\begin{array}{l}\mathbf{S} \\
(\mathbf{P E} / \mathrm{M})\end{array}$ & $\begin{array}{l}\text { Biyoiklim } \\
\text { Katı }\end{array}$ \\
\hline Eskişehir & 801 & 361,7 & 21,9 & $-0,4$ & 57,12 & 49,3 & 2,25 & $\begin{array}{l}\text { Yarı Kurak } \\
\text { Akdeniz }\end{array}$ \\
\hline
\end{tabular}

Araştırma alanının tanımı, coğrafi konum ve topografya bilgilerinin açıklanmasında Davis, P. H., 1965-1988 ve Eskişehir Orman Toprakları ve Ekoloji Araştırmaları Enstitüsü Müdürlüğü, Toprak Su, Verileri, 1984 kaynaklarından yararlanılarak düzenlenmiştir. Bu bölgenin bitki örtüsünün tespit edilmesi ile hem Eskişehir'in hem de Türkiye'nin florasına katkı sağlanılması amaçlanılmıştır.

\section{Materyal ve Metot}

Araştırmamızın materyalini oluşturan bitki örnekleri, 2005-2007 yıllarında, Şubat ayı başlarından Kasım ayı sonlarına kadar değişik zamanlarda araziye gidilerek toplanmıştır. Arazi çalışmaları sırasında toplanan bitki örneklerinin lokaliteleri ve habitatları tespit edilerek bazı bitkilerin fotoğrafları çekilmiştir. Toplanan bitkilerin teşhislerinde başta, Flora of Turkey and the East Aegean Islands (Davis, 1965-1988) olmak üzere, Türkiye Sulak Alan Bitki Örtüsü(Seçmen ve Leblebici, 1996), Ağaçlar ve Çalılar (Ocak, 2007), Botanik Kılavuzu (Baytop, 2005), Başkentin Doğal Bitkileri (Erik, 1998), Türkçe Bitki Adları Sözcüğü (Baytop, 1994) gibi eserlerden faydalanılmıştır. Araştırma alanında tespit edilen bitki türlerinin listesi ekte verilmiştir. Tür, cins ve familyaların verilişinde Türkiye florasındaki sisteme uyulmuştur. Makalede kullanılan kısaltmalar aşağıdaki gibi yapılmıştır.

$\begin{array}{ll}\text { E.Medit. } & \text { :East Mediterranean } \\ \text { Ir.-Tur. } & \text { : Irano-Turanian } \\ \text { Euro-Sib. } & \text { : Euro-Siberian } \\ \text { LR(Ic) } & \text { : En az endişe verici } \\ \text { LR(cd) } & \text { : Koruma önlemi gerektiren } \\ \text { LR(nt) } & \text { : Tehdit altına girebilir } \\ \text { VU } & \text { : Zarar görebilir }\end{array}$

\section{Bulgular}

\subsection{Bitki Toplanan istasyonlar}

1. B3 Eskişehir: Büyükyayla köyü, ormanlık alanlar, $1100 \mathrm{~m}$

2. B3 Eskişehir: Büyükyayla yol ayırımı, ağaçlandırma sahası, $1050 \mathrm{~m}$.

3. B3 Eskişehir: Karaören göleti ve çevresi açık anlar, $1100 \mathrm{~m}$.

4. B3 Eskişehir: Büyükyayla köyü çevresi, orman altı, $1320 \mathrm{~m}$.

5. B3 Eskişehir: Büyükyayla köyü üstü, Zöhre uslu çeşmesi ve çevresi, $1230 \mathrm{~m}$.

6. B3 Eskişehir: Büyükyayla köyü, açık çayırlık alanlar, $1050 \mathrm{~m}$.

7. B3 Eskişehir: Büyükyayla köyü mezarlık içi, 1050 $\mathrm{m}$.

8. B3 Eskişehir: Gökbahçe köyü yolu üzeri, tarlalık alanlar, $1010 \mathrm{~m}$.

9. B3 Eskişehir: Kümbet-Büyükyayla arası yol ayırımı, yol kenarları, $1030 \mathrm{~m}$.

10.B3 Eskişehir: Gökbahçe köyü yolu üzeri, tarlalık alanlar, $1010 \mathrm{~m}$.

11.B3 Eskişehir: Karaören göleti ve çevresi açık alanlar, $1100 \mathrm{~m}$.

12.B3 Eskişehir: Kümbet-Büyükyayla arası yol ayırımı, yol kenarları, $1030 \mathrm{~m}$.

13.B3 Eskişehir: Büyükyayla ovası, sulak alanlar, $1070 \mathrm{~m}$

14.B3 Eskişehir: Gökbahçe köyü yolu üzeri, tarlalık alanlar, $1010 \mathrm{~m}$.

15.B3 Eskişehir: Türkmen dağları, Sarıcaova yolu üzeri, ormanlık alanlar, $1100 \mathrm{~m}$.

16.B3 Eskişehir: Türkmen dağları, şelale yolu üzeri, ormanlık alanlar, $1250 \mathrm{~m}$.

17.B3 Eskişehir: Büyükyayla köyü, açık çayırlık alanlar, $1050 \mathrm{~m}$.

18.B3 Eskişehir: Büyükyayla köyü orman üstü, orman yolu 2. km., $1200 \mathrm{~m}$.

19.B3 Eskişehir: Büyükyayla köyü ormanlık alanlar, $1100 \mathrm{~m}$ 
20.B3 Eskişehir: Karaören göleti ve çevresi açık alanlar, $1100 \mathrm{~m}$.

21.B3 Eskişehir: Büyükyayla köyü, açık çayırlık alanlar, $1050 \mathrm{~m}$.

22.B3 Eskişehir: Kümbet-Büyükyayla arası yol ayırımı, yol kenarları, $1030 \mathrm{~m}$.

23.B3 Eskişehir: Karaören göleti ve çevresi açık alanlar, $1100 \mathrm{~m}$.

24.B3 Eskişehir: Türkmen dağları, Sarıcaova yolu üzeri 4 km., Orman altı, $1150 \mathrm{~m}$.

25.B3 Eskişehir: Büyükyayla yol ayırımı, ağaçlandırma sahası, $1050 \mathrm{~m}$.

26.B3 Eskişehir: Karaören göleti ve çevresi açık alanlar, $1100 \mathrm{~m}$.

27.B3 Eskişehir: Büyükyayla köyü üstü, Zöhre uslu çeşmesi ve çevresi, $1230 \mathrm{~m}$,

28.B3 Eskişehir: Büyükyayla köyü, açık çayırlık alanlar1050 m.

29.B3 Eskişehir: Kümbet-Büyükyayla arası yol ayırımı, yol kenarları, $1030 \mathrm{~m}$.

30.B3 Eskişehir: Gökbahçe köyü yolu üzeri, tarlalık alanlar, $1010 \mathrm{~m}$.

31.B3 Eskişehir: Türkmen dağları, şelale yolu üzeri, ormanlık alanlar, $1250 \mathrm{~m}$.

32.B3 Eskişehir: Türkmen dağları, Sarıcaova yolu üzeri, ormanlık alanlar, $1100 \mathrm{~m}$.

33.B3 Eskişehir: Türkmen dağları, Kocakavak tepesi, ormanlık alanlar, $1450 \mathrm{~m}$.

34.B3 Eskişehir: Türkmen dağları, şelale yolu üzeri, ormanlık alanlar, $1250 \mathrm{~m}$.

35.B3 Eskişehir: Büyükyayla ovası, sulak alanlar, $1070 \mathrm{~m}$.

36.B3 Eskişehir: Büyükyayla köyü, açık çayırlık alanlar $1050 \mathrm{~m}$.

37.B3 Eskişehir: Kümbet-Büyükyayla arası yol ayırımı, yol kenarları, $1030 \mathrm{~m}$.

38.B3 Eskişehir: Karaören göleti ve çevresi açık alanlar, $1100 \mathrm{~m}$.

39.B3 Eskişehir: Büyükyayla köyü çevresi, orman altı, $1320 \mathrm{~m}$.

40.B3 Eskişehir: Büyükyayla köyü çevresi, orman altı, $1320 \mathrm{~m}$.

41.B3 Eskişehir: Kümbet-Büyükyayla arası yol ayırımı, yol kenarları, $1030 \mathrm{~m}$.

42.B3 Eskişehir: Büyükyayla yol ayırımı, ağaçlandırma sahası, $1050 \mathrm{~m}$.

43.B3 Eskişehir: Büyükyayla köyü ormanlık alanlar, $1100 \mathrm{~m}$.

44.B3 Eskişehir: Büyükyayla yol ayırımı, ağaçlandırma sahası, $1050 \mathrm{~m}$.

45.B3 Eskişehir: Büyükyayla köyü mezarlık içi, 1050 $\mathrm{m}$.

46.B3 Eskişehir: Kümbet-Büyükyayla arası yol ayırımı, yol kenarları, $1030 \mathrm{~m}$.

47.B3 Eskişehir: Karaören göleti, Pinus nigra altı, $1050 \mathrm{~m}$

48.B3 Eskişehir: Büyükyayla köyü üstü, Zöhre uslu çeşmesi ve çevresi, $1230 \mathrm{~m}$.

49.B3 Eskişehir: Büyükyayla köyü, açık çayırlık alanlar1050 m.

50.B3 Eskişehir: Büyükyayla köyü ormanlık alanlar, $1100 \mathrm{~m}$.

51.B3 Eskişehir: Karaören göleti ve çevresi açık alanlar, $1100 \mathrm{~m}$

52.B3 Eskişehir: Büyükyayla yol ayırımı, ağaçlandırma sahası, $1050 \mathrm{~m}$.

53.B3 Eskişehir: Büyükyayla köyü orman üstü, orman yolu 2. km., $1200 \mathrm{~m}$.

54.B3 Eskişehir: Büyükyayla köyü orman üstü, orman yolu 2. km., $1200 \mathrm{~m}$.

55.B3 Eskişehir: Karaören göleti, Pinus nigra altı, $1050 \mathrm{~m}$.

56.B3 Eskişehir: Karaören göleti ve çevresi açık alanlar, $1100 \mathrm{~m}$.

57.B3 Eskişehir: Büyükyayla köyü orman üstü, orman yolu 2. km., 1200 m.

58.B3 Eskişehir: Karaören göleti ve çevresi açık alanlar, $1100 \mathrm{~m}$.

59.B3 Eskişehir: Türkmen dağları, Sarıcaova yolu üzeri, ormanlık alanlar, $1100 \mathrm{~m}$.

60.B3 Eskişehir: Büyükyayla ovası, sulak alanlar, $1070 \mathrm{~m}$.

61.B3 Eskişehir: Gökbahçe köyü yolu üzeri, tarlalık alanlar, $1010 \mathrm{~m}$.

62.B3 Eskişehir: Büyükyayla-Fethiye arası, orman yolu, Pinus nigra altı, $1050 \mathrm{~m}$.

63. B3 Eskişehir: Türkmen dağları, şelale yolu üzeri, ormanlık alanlar, $1250 \mathrm{~m}$.

64.B3 Eskişehir: Büyükyayla ovası, sulak alanlar, $1070 \mathrm{~m}$.

65.B3 Eskişehir: Büyükyayla köyü üstü, Zöhre uslu çeşmesi ve çevresi, $1230 \mathrm{~m}$.

66.B3 Eskişehir: Türkmen dağları, Sarıcaova yolu üzeri $4 \mathrm{~km}$, Orman altı, $1150 \mathrm{~m}$.

67.B3 Eskişehir: Karaören göleti ve çevresi açık alanlar, $1100 \mathrm{~m}$.

68. Eskişehir: Karaören göleti, Pinus nigra altı, 1050 $\mathrm{m}$.

69.B3 Eskişehir: Kümbet-Büyükyayla arası yol ayırımı, yol kenarları, $1030 \mathrm{~m}$.

70.B3 Eskişehir: Türkmen dağları, Kocakavak tepesi, ormanlık alanlar, $1450 \mathrm{~m}$.

71.B3 Eskişehir: Büyükyayla köyü orman üstü, orman yolu 2. km., $1200 \mathrm{~m}$.

72.B3 Eskişehir: Türkmen dağları, Karakale tepesi, ormanlık alanlar, $1320 \mathrm{~m}$. 


\section{Floristik Liste}

Spermatophyta

Gymnospermae

Pinaceae

Pinus nigra J.F. Arnold. subsp. nigra var. caramanica (Loudon) Rehder, 10.03.06,S.A.: 001.

Cupressaceae

Juniperus oxycedrus L.subsp. oxycedrus, 20.04.06, S.A.:004.

Angiospermae

Dicotyledonae

Ranunculaceae

Nigella elata Boiss., 12.05.06, S.A.:005, Geniş Yayılışlı.

Delphinium venulosum Boiss., 24.05.06, S.A.:006, Endemik, İran Turan elementi.

Consolida orientalis (Gay) Schrod., 07.06.06, S.A.:007, Geniş Yayılışlı.

Consolida regalis S. F. Gray subsp. paniculata (Host) Soo, 07.06.06, S.A.: 008, Geniş Yayılışlı.

Adonis aestivalis L. subsp. aestivalis, 12.05.06, S.A.: 009, Geniş Yayılışlı.

Adonis flammea Jack, 12.05.06, S.A.: 010, Geniş Yayılışlı.

Ranunculus constantinopolitanus (DC.) D'Urv, 12.05.06, S.A.: 011, Geniş Yayılışlı.

Ranunculus arvensis L., 07.06.06, S.A.: 012, Geniş Yayılışlı.

\section{Papaveraceae}

*Papaver somniferum L., 24.05.06, S.A.: 013, Geniş Yayılışlı.

Papaver rhoeas L., 24.05.06, S.A.: 014, Geniş Yayılışlı.

Papaver dubium L., 24.05.06, S.A.: 015, Geniş Yayılışlı.

Hypecoum procumbens L., 07.06.06, S.A.: 016,Geniş Yayılışlı.

\section{Brassicaceae}

Brassica elongata Ehrh., 12.05.06, S.A.: 017, Geniş Yayılışlı.

Diplotaxis tenuifolia (L.) DC, 07.06.06, S.A.: 018, Geniş Yayılışlı.

Cardaria draba (L.) Desv subsp. draba, 11.07.06, S.A.: 019, Geniş Yayılışı.

Thlaspi arvense L., 11.07.06, S.A.: 020, Geniş Yayılışlı.

Capsella bursa-pastoris (L.) Medik, 24.05.06, S.A.:021, Geniş Yayılışlı.

Boreava orientalis Jaub \& Spach, 24.05.06,S.A.: 022, Geniş Yayılışlı.

Fibigia clypeata (L.) Medik, 24.05.06, S.A.: 023, Geniş Yayılışlı.
Alyssum dasycarpum Stephen ex Willo, 11.07.06, S.A.:024, Geniş Yayılışlı.

Alyssum strigosum Banks et Sol, 11.07.06, S.A.:025, Geniş Yayılışlı.

Clypeola jonthlaspi L., 11.07.06, S.A.: 026, Geniş Yayılışlı.

Erophila verna (L.) Chevall subsp. verna, 24.05.06, S.A.: 027, Geniş Yayılışlı.

Arabis caucasica Willd. subsp. caucasica, 24.05.06, S.A.: 028, Geniş Yayılışlı.

Aubrieta deltoidea (L.) DC., 12.05.06, S.A.: 029, Geniş Yayılışlı.

Hesperis balansae Fourn, 12.05.06, S.A.: 030, Endemik, İran-Turan elementi.

Alliaria petiolata ( Bieb.) Cavara et Grande, 12.05.06, S.A.: 031, Geniş Yayılışlı.

Sisymbrium altissimum L., 07.06.06, S.A.:032, Geniş Yayılışlı.

Descurainia sophia (L.) Webb ex Prantl, 24.05.06, S.A.:033, Geniş Yayılışlı.

Resedaceae

Reseda lutea L. var. Iutea, 07.06.06, S.A.:034, Geniş Yayılışlı.

Cistaceae

Helianthemum canum (L.) Bavmg, 24.05.06, S.A.:035, Geniş Yayılışlı.

Violaceae

Viola odorata L., 12.05.06, S.A.: 036, Geniş Yayılışlı. Viola kitaibeliana, 12.05.06, S.A.: 037, Geniş Yayılışlı.

Polygalaceae

Polygala anatolica Boiss. et Heldr., 07.06.06, S.A.:038, Geniş Yayılışlı.

Polygala vulgaris L.,07.06.06, S.A.: 039, Geniş Yayılışlı.

\section{Caryophyllaceae}

Stellaria media (L.) Vill. subsp. media, 07.06.06, S.A.: 040, Geniş Yayılışlı.

Holesteum umbellatum L. var. umbellatum, 07.06.06, S.A.: 041, Geniş Yayılışlı.

Dianthus zonatus Fenzl. var. zonatus, 11.07.06 S.A.: 042, Geniş Yayılışlı.

Silene italica (L.) Pers, 11.07.06, S.A.: 043, Geniş Yayılışlı.

Silene lydia L. Boiss., 12.07.06, S.A.: 044, Geniş Yayılışlı.

Polygonaceae

Polygonum cognatum Meissn., 07.06.06, S.A.:045, Geniş Yayılışlı.

Rumex acetosella L.,15.08.06, S.A.: 046, Geniş Yayılışlı.

Rumex crispus L.,15.08.06, S.A.: 047, Geniş Yayılışlı. 


\section{Chenopodiaceae}

Beta vulgaris L., 07.06.06, S.A.: 048, Geniş Yayılışlı. Chenopodium botrys L., 07.06.06, S.A.: 049, Geniş Yayılışlı.

Chenopodium album L., 12.07.06, S.A.: 050, Geniş Yayılışlı.

\section{Amaranthaceae}

Amaranthus retroflexus L.,12.07.06, S.A.:051, Geniş Yayılışlı.

Hypericaceae

Hypericum confertum Choisy. subsp. confertum, 12.07.06, S.A.:052

Hypericum linarioides Bosse, 11.07.06, S.A.:053, Geniş Yayılışlı.

Hypericum orientale L.,07.06.06, S.A.:054

Hypericum montbretii Spach, 07.06.06, S.A.:055

Hypericum perforatum L.,24.05.06, S.A.:056

Malvaceae

Hibiscus trionum L.,24.05.06, S.A.:057, Geniş Yayılışlı.

Malva sylvestris L.,24.05.06, S.A.:058, Geniş Yayılışlı.

Alcea rosea L.,24.05.06, S.A.:059, Geniş Yayılışlı. Linaceae

Linum bienne Miller, 12.05.06, S.A.: 060, Akdeniz elementi.

\section{Geraniaceae}

Geranium rotundifolium L., 24.05.06, S.A.: 061, Geniş Yayılışlı.

Geranium asphodeloides subsp. sintenisii pyreanicum Burm. Fil., 24.05.06, S.A.: 062, Geniş Yayılışlı.

Erodium cicutarium (L.) L'Hérit. subsp. cicutarium (L.) L'Herit, 07.06.06, S.A.: 063, Geniş Yayılışlı.

Rutaceae

Haplophyllum thesioides (Fisch. ex DC.) G.Don, 12.07.06, S.A.: 064

Rhamnaceae

Paliurus spina-christi Miller, 15.08.06, S.A.: 065, Geniş yayılışlı.

Frangula alnus Miller subsp. alnus, 15.08.06, S.A.:066, Geniş yayılışlı.

Fabaceae

Chamaecytisus hirsutus (L.) Link, 12.05.06, S.A.:067, Geniş Yayılışlı.

Colutea cilicica Boiss. \& Bal., 20.04.06, S.A.:068, Geniş Yayılışlı.

Astragalus glycyphyllos L. subsp. glycyphylloides (DC.) Matthews, 07.06.06, S.A.: 069, Geniş Yayılışlı. Astragalus vulnerariae DC., 07.06.06, S.A.: 070, Endemik.
Astragalus angustifolius Lam., 07.06.06, S.A.: 071, Geniş Yayılışlı.

Vicia lathyroides L., 24.05.06, S.A.: 072

Vicia cracca L. subsp. stenophylla Vel, 24.05.06, S.A.: 073, Avrupa-Sibirya Elementi.

Lathyrus pratensis L., 12.05.06, S.A.: 074, AvrupaSibirya Elementi.

Lathyrus laxiflorus (Desf.) O. Kuntze subsp. laxiflorus (Desf.)O. Kuntze, 12.05.06, S.A.:075, Doğu Akdeniz Elementi.

Ononis spinosa L. subsp. antiquorum (L.) Briq, 07.06.06, S.A.: 076, Geniş Yayılışlı.

Trifolium repens L. var. repens, 12.05.06, S.A.:077, Geniş Yayılışlı.

Trifolium campestre Schreber, 12.05.06, S.A.:078, Geniş Yayılışlı.

Trifolium pratense L. var. pratense, 12.05.06, S.A.:079, Geniş Yayılışlı.

Trifolium arvense L. var. arvense, 20.04.06, S.A.:080, Geniş Yayılışlı.

Melilotus officinalis (L.) Desr., 12.05.06, S.A.:081, Doğu Akdeniz Elementi.

Trigonella cretica (L.) Boiss., 24.05.06, S.A.:082, Doğu Akdeniz Elementi.

Medicago Iupulina L., 24.05.06, S.A.:083, Geniş Yayılışlı.

Medicago sativa L. subsp. sativa L., 24.05.06, S.A.:084, Geniş Yayılışlı.

Coronilla varia L. subsp. varia L., 20.04.06, S.A.:085, Geniş Yayılışlı.

Hedysarum varium Willd., 24.05.06, S.A.: 086, İranTuran Elementi.

Onobrychis sativa, 12.05.06, S.A.: 087, Geniş Yayılışlı.

Onobrychis armena Boiss. \& Huet, 12.05.06, S.A.:088, Geniş Yayılışlı.

Rosaceae

Prunus spinosa L. supsp. Dasyphylla (Schur) Domin, 12.07.06, S.A.: 089, Avrupa-Sibirya Elementi.

Rubus sanctus Schreber, 24.05.06, S.A.: 090, Geniş Yayılışlı.

Potentilla reptans L., 11.07.06, S.A.: 091, Geniş Yayılışlı.

Fragaria vesca L., 07.06.06, , S.A.: 092, Geniş Yayılışlı.

Rosa pulverulenta Bieb., 07.06.06, S.A.: 093, Geniş Yayılışlı.

Rosa canina L., 12.05.06, S.A.: 094, Geniş Yayılışlı. Cydonia oblonga Miller, 12.05.06, S.A.: 095, Geniş Yayılışlı.

Malus sylvestris (L.) Miller subsp. orientalis (A. Uglitzkich) Browicz, 12.05.06, S.A.:096, Geniş Yayılışlı. 
Pyrus elaeagnifolia Pallas, 12.05.06, S.A.:097

\section{Onagraceae}

Epilobium hirsutum L., 12.05.06, S.A.:098, Geniş Yayılışlı.

Epilobium parviflorum Schreber, 07.06.06,

S.A.:099, Geniş Yayılışlı.

Cucurbitaceae

Ecballium elaterium (L.) A. Rich., 07.06.06, S.A.:100, Akdeniz Elementi.

\section{Crassulaceae}

Sedum amplexicaule DC. Subsp. tenuifolia (Sm.) Greuter , 24.05.06, S.A.: 101, Geniş Yayılışlı.

Sedum acre L., 24.05.06, , S.A.: 102, Geniş Yayılışlı.

\section{Apiaceae}

Eryngium campestre L. var. campestre (L.) Hudson.,07.06.06, S.A.: 103, Geniş Yayılışı.

Echinophora tenuifolia L. subsp. sibthorpiana (Guss.) Tutin., 07.06.06, S.A.: 104, Geniş Yayılışlı.

Anethum graveolens L., 07.06.06, S.A.: 105, Geniş Yayılışlı.

Conium maculatum L., 12.05.06, S.A.: 106, Geniş Yayılışlı.

Turgenia latifolia (L.) Hoffm., 11.07.06, S.A.: 107, Geniş Yayılışlı.

Daucus carota L., 07.06.06, S.A.: 108, Geniş Yayılışı.

\section{Araliaceae}

Hedera helix L, 24.05.06, S.A.: 109, Geniş yayılışlı. Dipsacaceae

Dipsacus laciniatus L., 11.07.06, S.A.: 110, Geniş Yayılışlı.

Scabiosa argentea L., 11.07.06, S.A.: 111, Geniş Yayılışlı.

\section{Asteraceae}

Xanthium spinosum L., 07.06.06, S.A.: 112, Geniş Yayılışı.

Xanthium strumarium L. subsp. strumarium, 11.07.06, S.A.: 113, Geniş Yayılışı.

Erigeron acer L. subsp. pycnotrichus (Vierh.) Grierson, 11.07.06, S.A.: 114, Geniş Yayılışlı.

Senecio pseudo-orientalis Schischkin, 12.07.06, S.A.: 115, İran-Turan Elementi.

Tussilago farfara L., 12.05.06, S.A.: 116, Geniş Yayılışlı, Avrupa-Sibirya Elementi.

Anthemis cretica L. subsp. anatolica (Boiss.) Grierson, 24.05.06, S.A.: 117, Geniş Yayılışlı.

Anthemis tinctoria L. var. tinctoria, 24.05.06, , S.A.:118, Geniş Yayılışı, Doğu Akdeniz Elementi.

Anthemis tinctoria L. var. pallida DC., 07.06.06, S.A.: 119, Geniş Yayılışı.

Achillea wilhelmsii C. Koch, 07.06.06, S.A.: 120, Geniş Yayılışlı.
Circium arvense (L.) Scop. subsp. vestitum (Wimmer \& Grab.) Petrak, 12.07.06, S.A.: 121, Geniş yayılışlı.

Cirsium vulgare (Savi) Ten., 12.07.06, S.A.: 122, Geniş yayılışlı.

Picnomon acarna (L.) Cass., 24.05.06, S.A.: 123, Geniş Yayılışıl, Akdeniz Elementi.

Carduus nutans L., 12.07.06, S.A.: 124, Geniş Yayılışlı.

Acroptilon repens (L.) DC., 12.07.06, S.A.: 125, Geniş Yayılışlı.

Centaurea solstitialis L. subsp. solstitialis, 12.07.06, S.A.: 126, Geniş Yayılışı, Doğu Akdeniz Elementi.

Centaurea urvillei DC. subsp. urvillei, 12.07.06, S.A.: 127, Doğu Akdeniz Elementi.

Centaurea iberica Trev. ex Sprengel, 12.07.06, S.A.:128, Geniş Yayılışlı.

Centaurea depressa Bieb., 12.07.06, S.A.:129, Geniş Yayılışlı.

Crupina crupinastrum (Moris) Vis., 07.06.06, S.A.:130, Geniş Yayılışlı.

Xeranthemum annuum L., 07.06.06, S.A.:131, Geniş Yayılışlı.

Echinops viscosus DC. subsp. bithynicus (Boiss.) Rech., 11.07.06, S.A.: 132, Geniş Yayılışlı.

Scolymus hispanicus L., 11.07.06, S.A.:133, Akdeniz Elementi.

Cichorium intybus L., 15.08.06, S.A.:134, Geniş Yayılışlı.

Tragopogon latifolius Boiss. var. angustifolius Boiss., 24.05.06, S.A.: 135, Geniş Yayılışı.

Leontodon crispus Vill. subsp. asper (Walds. Et Kit) Rohl. var asper Vill., 24.05.06, S.A.:136, Geniş Yayılışlı.

Sonchus asper (L.) Hill. subsp. glaucescens (Jordan) Ball, 24.05.06, S.A.:137, Geniş Yayılışlı.

Lactuca serriola L., 12.07.06, S.A.:138, Geniş Yayılışlı.

Taraxacum officinale Weber, 12.05.06, S.A.:139, Geniş Yayılışlı.

\section{Campanulaceae}

Campanula lyrata Lam. subsp. lyrata, 12.05.06, S.A.:140, Endemik, Geniş Yayılışlı.

Campanula rapunculoides $\mathrm{L}$. subsp. cordifolia (C. Koch) Damboldt , 24.05.06, S.A.: 141, Geniş Yayılışlı.

Campanula stricta L. var. stricta, 24.05.06, S.A.:142, Geniş Yayılışlı.

Primulaceae

Anagallis arvensis L. var. arvensis, 24.05.06, S.A.:143, Geniş Yayılışlı. 
Anagallis foemina Miller., 20.04.06, S.A.: 144, Geniş Yayılışlı.

Convolvulaceae

Convolvulus arvensis L., 24.05.06, S.A.:145, Geniş Yayılışlı.

Convolvulus lineatus L., 24.05.06, S.A.:146, Geniş Yayılışlı.

\section{Cuscutaceae}

Cuscuta campestris Yuncker, 07.06.06, S.A.:147.

Boraginaceae

Heliotropium europaeum L., 24.05.06, S.A.:148, Akdeniz Elementi.

Myosotis sicula Guss., 07.06.06, S.A.:149, Geniş Yayılışlı.

Echium italicum L., 24.05.06, S.A.:150, Geniş Yayılışlı.

Echium orientale L., 24.05.06, S.A.:151, Endemik, Öksin element.

Moltkia coerulea (Willd.) Lehm., 12.05.06, S.A.:152, İran-Turan Elementi.

Onosma armenum DC., 07.06.06, S.A.:153, Geniş Yayılışlı.

Onosma tauricum Pallas ex Willd. var. tauricum, 07.06.06, S.A.: 154, Geniş Yayılışlı.

Anchusa azurea Miller subsp. azurea, 24.05.06, S.A.: 155, Geniş Yayılışlı.

Alkanna orientalis (L.) Boiss. var. leucantha (Bornm.) Hub.-Mor., 24.05.06, S.A.: 156, Endemik, İran-Turan elementi.

\section{Solanaceae}

Hyocyamus niger L., 12.05.06, S.A.: 157, Geniş Yayılışlı.

\section{Scrophulariaceae}

Verbascum flavidum (Boiss.) Freyn \& Bornm, 12.07.06, S.A.: 158, Avr.-Sib. Elementi.

Verbascum bombyciferum Boiss., 12.07.06, S.A.:159, Endemik., Avr.-Sib. Elementi.

Scrophularia scopolii (Hoppe ex) Pers. var. scopolii (Hoppe ex), 07.06.06, S.A.: 160, Geniş Yayılışlı.

Linaria corifolia Desf., 07.06.06, S.A.: 161, Endemik, İran-Turan Elementi.

Veronica serpyllifolia L., 24.05.06, S.A.: 162, Geniş Yayılışlı.

Veronica officinalis L., 24.05.06, S.A.: 163, Endemik.

Orobanchaceae

Orobanche minor Sm., 24.05.06, S.A.: 164.

Orobanche mutelii F. Schultz, 24.05.06, S.A.: 165

Acanthaceae

Acanthus hirsutus Boiss., 07.06.06, S.A.: 166, Endemik, Geniş Yayılışlı.
Lamiaceae

Ajuga chamaepitys (L.) Schreber subsp. chia (Schreber) Arcangeli var. chia, 24.05.06, S.A.: 167, Geniş Yayılışlı.

Scutellaria velenouskyi Fil, 12.05.06, S.A.: 168, Doğu Akdeniz elementi.

Lamium garganicum subsp. laevigatum Arganceli, 24.05.06, S.A.: 169, Geniş Yayılışlı.

Lamium amplexicaule L., 24.05.06, S.A.: 170, Geniş Yayılışlı, Avrupa-Sibirya Elementi.

Stachys byzantina C. Koch, 20.04.06, S.A.: 171, Geniş Yayılışlı.

Nepeta italica L. Supsp. İtalica., 24.05.06, S.A.: 172, Geniş Yayılışlı.

Thymus longicaulis C. Presl subsp. Iongicaulis C. Presl var. subisophyllus (Borbas) Jalas, 24.05.06, S.A.: 173, Geniş Yayılışlı.

Thymbra spicata L. var. spicata, 24.05.06, S.A.:174, Geniş Yayılışlı.

Mentha longifolia (L.) Hudson subsp. typhoides (Briq.) Harley var. typhoides, 24.05.06, S.A.: 175, Geniş Yayılışlı.

Ziziphora taurica Bieb. subsp. taurica, 07.06.06, S.A.: 176, Geniş Yayılışlı, İran-Turan Elementi.

Salvia virgata Jacq., 24.05.06, S.A.: 177, Geniş Yayılışlı.

Salvia wiedemannii Boiss., 24.05.06, S.A.: 178, Endemik, İran-Turan elementi.

Plumbagınaceae

Acantholimon acerosum (Willd.) Boiss. subsp. acerosum (Willd.) Boiss, 12.07.06, S.A.: 179, Geniş Yayılışlı.

Plantagınaceae

Plantago major L. subsp. intermedia (Gilib.) Lange, 07.06.06, S.A.: 180, Geniş Yayılışlı.

Loranthaceae

Viscum album L. subsp. album, 07.06.06, S.A.: 181

Aristolochiaceae

Aristolochia pallida, 12.05.06, S.A.: 182, Geniş Yayılışlı.

Euphorbiaceae

Euphorbia helioscopia L., 12.05.06, S.A.: 183, Geniş yayılışlı.

Euphorbia falcata L. subsp. falcata var. galilaea (Boiss.) Boiss., 24.05.06, S.A.: 184, Doğu Akdeniz Elementi.

Urticaceae

Urtica dioica L., 07.06.06, S.A.: 185, Geniş yayılışlı.

Platanaceae

Platanus orientalis L., 15.08.06, S.A.: 186, Geniş Yayılışlı. 


\section{Fagaceae}

Quercus infectoria Olivier subsp. boissieri (Reuter) O. Sch., 15.08.06, S.A.: 187, Geniş Yayılışlı.

Quercus petrea ( Mattuschka ) Liebl. subsp. iberica (Steven ex Bieb.) Krassiln., 15.08.06, S.A.:188, Geniş Yayılışlı.

Salicaceae

Salix alba L., 12.07.06, S.A.:189, Geniş Yayılışlı, Avrupa-Sibirya Elementi.

Populus nigra L. subsp. nigra, 12.07.06, S.A.:190, Avrupa-Sibirya Elementi.

Rubiaceae

Asperula arvensis L., 24.05.06, S.A.:191, Geniş Yayılışlı, Akdeniz Elementi.

Galium album Miller subsp. pycnotrichum (H.Braun) Krendl, 24.05.06, S.A.: 192, Geniş yayılışlı. Galium verum L. subsp. verum, 24.05.06, S.A.:193, Avrupa-Sibirya Elementi.

\section{Monocotyledonae}

Liliacaeae

Polygonatum orientale Desf., 20.04.06, S.A.:194, Geniş yayılışlı.

Asphodeline lutea (L.) Reichb., 24.05.06, S.A.:195, Geniş yayılışlı.

Allium cupani L. Rafin. subsp. hirtovaginatum (Kunth) Stearn, 12.07.06, S.A.:196, Geniş Yayılışlı.

Allium olympicum Boiss., 12.07.06,S.A.:197, Endemik, Öksin Elementi.

Ornithogalum ulophyllum Hand-Mazz, 20.04.06, S.A.:198, Geniş Yayılışlı.

Ornithogalum fimbriatum Willd., 20.04.06, S.A.:199, Geniş Yayılışlı.

Ornithogalum pyrenaicum L., 07.06.06, S.A.:200, Geniş Yayılışlı.

Ornithogalum sigmoideum Freyn \& Sint., 25.03.06, S.A.:201, Geniş Yayılışlı.

Ornithogalum oligophyllum E.D. Clarke, 12.05.06, S.A.:202, Geniş Yayılışlı.

Muscari neglectum Guss., 10.03.06, S.A.:203, Geniş Yayılışlı, Crp.

Muscari armeniacum Leichtlin ex Baker, 12.05.06, S.A.: 204, Geniş Yayılışlı.

Muscari latifolium Kirk, 12.05.06, S.A.:205, Endemik, Doğu Akdeniz Elementi.

Fritillaria pinardii Boiss., 12.05.06, S.A.:206, Geniş Yayılışlı.

Gagea villosa (Bieb.) Duby var. villosa (Bieb.) duby, 12.05.06, S.A.: 207, Geniş Yayılışlı.

Colchicum szovitsii Fisch.\& Mey., 10.03.06, S.A.:208, İran-Turan Elementi.

Merendera attica (Spruner) Boiss. \& Spruner, 10.03.06, S.A.: 209, Doğu Akdeniz Elementi.
Iridaceae

Iris orientalis Mill., 24.05.06, S.A.:210, Doğu Akdeniz Elementi.

Crocus ancyrensis (Herbert) Maw, 25.03.06, S.A.:211, Endemik, İran-Turan Elementi.

Crocus chrysanthus (Herbert) Herbert, 25.03.06, S.A.:212, Geniş Yayılışlı.

Crocus danfordiae Maw, 25.03.06, S.A.:213, Endemik.

\section{Orchidaceae}

Cephalanthera rubra (L.) L. C. M. Richard, 20.04.06, S.A.: 214, Geniş Yayılışlı.

Epipactis condensata Boiss. Ex D. P. Young, 20.04.06, S.A.:215, Geniş Yayılışlı.

Orchis anatolica Boiss., 25.03.06, S.A.:216, Geniş Yayılışlı.

Dactylorhiza iberica (Bieb. ex Willd.) Soo, 12.05.06, S.A.:217, , Geniş Yayılışlı.

Typhaceae

Typha angustifolia L.,15.08.06, S.A.:218, Geniş Yayılışlı.

Juncaceae

Juncus inflexus L.,15.08.06, S.A.:219, Geniş Yayılışlı. Cyperaceae

Eleocharis palustris (L.) Roemer\&Schultes, 15.08.06, S.A.:220, Geniş Yayılışlı.

Poaceae

Aegilops geniculata Roth., 11.07.06, S.A.:221, Geniş Yayılışlı.

Aegilops triuncialis L. supsp. triuncialis L.,11.07.06, S.A.:222, Geniş Yayılışlı.

Triticum aestivum L., 11.07.06, S.A.:223, Geniş Yayılışlı.

Hordeum murinum L. var. glaucum (Steudel) Tzvelev, 11.07.06, S.A.:224

Bromus rubens, 11.07.06, S.A.:225

Bromus hordeaceus subsp. hordeaceus, 11.07.06, S.A.:226

Avena barbata Pott ex link. subsp. barbata Pott ex link., 15.08.06, S.A.:227, Geniş Yayılışlı, Akdeniz Elementi.

Phleum pratense L.,15.08.06, S.A.:228, Geniş Yayılışlı, Avrupa-Sibirya Elementi.

Poa bulbosa L., 11.07.06, S.A.:229, Geniş Yayılışlı.

Melica ciliata L. subsp. ciliata, 07.06.06, S.A.:230, Geniş Yayılışlı.

Phragmites australis (Cav.) Trin. ex Steudel, 11.07.06, S.A.:231, Geniş Yayılışlı, Avrupa-Sibirya Elementi.

Cynodon dactylon (L.) Pers var. dactylon (L.)Pers, 11.07.06, S.A.: 232, Geniş Yayılışlı. 


\section{Tartışma ve Sonuç}

Bu çalışmada 55 familya'ya ait 170 cins ve 230 tür ve türaltı takson, , tespit edilmiştir. Tespit edilen taksonlardan 2'si Gymnospermae alt bölümüne, diğer 228 takson ise Angiospermae alt bölümüne aittir. Bunlarında 39'u Monocotyledonae, 191 takson ise Dicotyledonae sınıfına aittir.

Araştırma alanından toplanan taksonlarının ait oldukları fitocoğrafik bölgelere göre dağılış oranı şöyledir: Avrupa-Sibirya \% 5.2, İran -Turan \% 4.78, Akdeniz \% 6.96, belirlenemeyen \% 83'dür. Buna göre oranların birbirine yakın olması araştırma alanının söz konusu üç fitocoğrafik bölgenin etkisi altında olduğunu göstermektedir.

Araştırma alanından tespit edilen taksonların fitocoğrafik bölge elementleri oranlarının yakın bölgelerdeki çalışmalar ile karşılaştırılmıştır.

Tablo 2. Araştırma Alanına Ait Fitocoğrafik Bölge Elementlerinin Yakın Bölgelerde Yapılan Çalışmalar ile Karşılaştırılması (\%)

\begin{tabular}{|l|c|c|c|c|c|c|}
\hline $\begin{array}{l}\text { Fitocoğrafik } \\
\text { bölge }\end{array}$ & I & II & III & IV & V & VI \\
\hline İran-Turan & 4.78 & 16.46 & 10.06 & 18 & 22.9 & 4.9 \\
\hline Akdeniz & 6.96 & 7.52 & 14.6 & 25 & 7.5 & 16.5 \\
\hline Avrupa-Sibirya & 5.2 & 7.32 & 7.87 & 20 & 4.9 & 7.7 \\
\hline Bilinmeyenler & 83 & 68.70 & 67.47 & 37 & 64 & 70.7 \\
\hline
\end{tabular}

I- Büyükyayla (Eskişehir) Florası (Arı, 2007)

II- Mihalıççık (Eskişehir) ilıçesinin Florası (Özaydın ve Yücel, 2004)

III- Gülümbe Dağı Flora ve Vejetasyonu (Ocak 2001)

IV- Sündiken Dağları (Eskişehir) Vejetasyonunun Sosyolojik ve Ekolojik Yönden Araştırılması (Ekim, 1991)

V- Demirlik ve Kulaksız (Kütahya) Dağları Florası (Akçiçek, 2002)

VI- Simav Dağı Florası (Yayıntaş, 1985)

Tablo 3. Araştırma Alanı Endemizm Oranının Yakın Bölge Çalışmaları İle Karşılaştırılması

\begin{tabular}{|c|c|c|c|c|c|c|}
\hline \multirow{2}{*}{$\begin{array}{c}\text { Endemizm } \\
\text { Oranı (\%) }\end{array}$} & \multicolumn{6}{|c|}{ Araştırma Alanları } \\
\cline { 2 - 7 } & $\begin{array}{c}6.9 \\
6\end{array}$ & 12.60 & 7 & 10 & 18.3 & 9.4 \\
\hline
\end{tabular}

Araştırma alanında 16 adet endemik takson belirlenmiştir. Endemizm oranı \% 6.96'dır. Çalışmamız endemizm oranı bakımından yakın bölgelerde yapılan floristik çalışmalarla karşılaştırıldığında Ocak ve Tokur'un yapmış olduğu çalışmaya yakın olduğu bulunmuştur.

Tablo 4. Araştırma Alanında En Fazla Takson İçeren Familyaların Yakın Bölgelerdeki Floristik Çalışmalar ile Karşılaştırılması (\%)

\begin{tabular}{|l|c|c|c|c|c|c|}
\hline Familya & I & II & III & IV & V & VI \\
\hline Asteraceae & 12.06 & $\mathbf{1 1 . 5 8}$ & 8.78 & 9.70 & 12.95 & 11.62 \\
\hline Brassicaceae & 7.32 & 3.92 & 7.44 & 4.92 & 8.30 & 6.00 \\
\hline Poaceae & 5.17 & 8.41 & 7.65 & 3.73 & 7.30 & 5.81 \\
\hline Liliaceae & 6.89 & 3.92 & 2.84 & ----- & 3.98 & 3.48 \\
\hline Lamiaceae & 5.17 & 8.41 & 8.53 & 5.97 & 8.63 & 6.58 \\
\hline Fabaceae & 9.48 & 9.71 & 8.96 & 9.55 & 8.97 & 13.37 \\
\hline Boraginaceae & 3.87 & 2.99 & 3.50 & ---- & 3.32 & 4.84 \\
\hline Caryophyllaceae & 2.15 & 2.42 & 2.84 & 3.28 & --- & --- \\
\hline Apiaceae & 2.58 & 2.61 & 3.50 & 4.47 & 4.98 & 2.51 \\
\hline
\end{tabular}

Araştırma sonuçları yakın çevrede yapılan diğer flora çalışmaları ile karşılaştırıımıştır (Tablo 4). Karşıllaştırma sonucunda özellikle Asteraceae, Brassicaceae, Poaceae ve Liliaceae familyalarının diğer bölgelerde yapılan floristik çalışmalara göre daha yüksek oranda taksonla temsil edildiği anlaşılmaktadır.

Diğer familyalardaki takson sayısının yakın bölgelerde yapılan araştırma sonuçları ile benzerlik göstermesi, çalışma alanlarının benzer iklimsel faktörlerin etkisinde olduğunu düşündürmektedir.

\section{Kaynaklar}

Akçiçek, E., Demirlik ve Kulaksız(Kütahya) Dağları Florası, Ot Sistematik Botanik Dergisi, 2002;9(3):19-46.

Akman, Y., İklim ve Biyoiklim (Biyoiklim Metodları ve Türkiye iklimleri), Mühendislik Serisi: 103, Kasım, 1990.

Baytop, T., Türkçe Bitki Adları Sözlüğü, Türk Dil Kurumu Yayınları, 578, Ankara, 1994.

Davis, P. H., (ed) 1965-1988, Flora of Turkey and The Easr Aegean Islands, Vol. 1-10 (suppl.), Edinburgh Ünv. Pres., Edinburgh.

Ekim, T., Sündiken Dağları (Eskişehir) Vejetasyonunun Sosyolojik ve Ekolojik Yönden Araştırılması, 1991.

Erik, S., AKAYDIN,G., GÖKTAÇ, A., Başkentin Doğal Bitkileri, Ankara Üniversitesi Basımevi, Ankara, 1998. 
Ocak, A., "Eskişehir, Çatacık Florası II", Cetemenler Dijital Basım-Eskişehir, 2007.

Ocak, A., Tokur, S. 2000. The Flora of Gülümbe Dağı (Bilecik-Turkey). Turkish Journal of Botany. 24: 121141.

Toprak su., Eskişehir Orman Toprakları ve Ekoloji Araştırmaları Enstitüsü Müdürlüğü, Toprak Su, Verileri, Eskişehir, 1984.

Yayıntaş, A., (Simav Dağı) Florası, Doğa Bilim Dergisi, 1985;9(2):388-418. 